\title{
Critical Appraisal of Experimental Radiation Modalities for Malignant Astrocytomas
}

\author{
N.J. Laperriere
}

\begin{abstract}
The management of patients with supratentorial malignant astrocytomas has remained a major problem. Patients continue to die from a lack of local control in $90 \%$ of cases despite an improvement of median survival seen with the use of postoperative radiation therapy. Because of this, there has been considerable interest in exploring novel ways of possibly improving results. This paper reviews the rationale and clinical results with the use of altered fractionation schemes, brachytherapy, radiation sensitizers, hyperthermia, particle therapy, and radiosurgery in the treatment of these patients. Currently, there is no demonstrated advantage with the use of these experimental modalities in the initial management of patients. There would appear to be some benefit for selected patients who are treated with brachytherapy at recurrence, but its efficacy as part of initial management remains to be determined in ongoing randomized prospective trials.
\end{abstract}

RÉSUMÉ: Évaluation critique des modes expérimentaux d'irradiation dans l'astrocytome malin Le traitement des patients porteurs d'astrocytomes malins sus-tentoriels demeure un problème majeur. Ces patients continuent de mourir par manque de contrôle de la tumeur locale dans $90 \%$ de cas, malgré une amélioration de la survie médiane constatée avec l'emploi de la radiothérapie postopératoire. C'est ce qui a suscité un vif intérêt dans l'exploration de nouveaux moyens pouvant possiblement améliorer les résultats. Cet article revoit la justification et les résultats cliniques obtenues avec l'utilisation de plans modifiés de fractionnement de la radiothérapie, de brachythérapie, de radio-sensibilisateurs, d'hyperthermie, de thérapie particulaire, et de radiochirurgie dans le traitement de ces patients. Il n'y a actuellement aucune preuve que l'utilisation de ces modalités thérapeutiques expérimentales soit avantageuse dans la conduite initiale du traitement chez ces patients. Actuellement, il semblerait y avoir un réel bénéfice pour un groupe sélectionné de patients qui sont traités par brachythérapie au moment d'une récidive. Cependant, l'efficacité de cette modalité thérapeutique dans la conduite initiale du traitement reste à déterminer par des études prospectives randomisées présentement encours.

Can. J. Neurol. Sci. 1990; 17:199-208

Malignant astrocytomas account for approximately $55 \%$ of primary brain neoplasms in adults, with an anticipated 2 year survival of 5\%. ${ }^{1}$ The disease is seen predominently in the 40 to 70 year age group. With the use of surgery alone, the median survival is 14 weeks. $^{2}$ When surgery is followed by external beam radiation, there is an increase in the median survival to 35 48 weeks. 2,3 The addition of systemic chemotherapy has only been of modest benefit. ${ }^{4}$

As opposed to most other malignancies, malignant astrocytomas are fatal as a result of uncontrolled local growth. 5,6 Cerebral spinal fluid seeding occurs very infrequently, and extra-neural metastases are quite uncommon. ${ }^{7}$ For these reasons, there has been considerable interest in exploring the use of experimental radiation modalities in the management of this disease. This paper reviews results to date with the use of altered fractionation, brachytherapy, radiation sensitizers, hyperthermia, particle therapy, and radiosurgery in patients with malignant astrocytomas.

\section{Altered Fractionation}

Conventional external radiation is delivered once daily Monday to Friday with no treatment on weekends or holidays. This method of delivering radiation was developed empirically and was influenced predominently by clinical observations of cures and economic feasibility. ${ }^{8}$ The two alternate methods of fractionation that have been investigated are accelerated fractionation and hyperfractionation.

\section{Accelerated Fractionation}

Accelerated fractionation is achieved when the overall treatment time is shortened. This is achieved with two or three fractions per day. The fraction size and total dose is similar to conventionally delivered radiation. The potential advantage of this approach is to decrease the time available for repopulation of the malignant clonogenic cells during treatment. There would also be an increased effect on acutely responding normal tissues, but there would not be an increase in late reacting tissues as

From the Department of Radiation Oncology, Princess Margaret Hospital, Toronto

Reprint requests to: N.J. Laperriere, M.D., Department of Radiation Oncology, Princess Margaret Hospital, 500 Sherbourne St., Toronto,

Ontario, Canada M4X $1 \mathrm{~K} 9$ 
long as the interval between fractions would be long enough to allow for repair of sublethal injury.

Simpson and Platts 9 reported their results with 9 separate fractionation schemes to which patients with glioblastoma multiforme were randomized in sequential groups of three. This involved a total of 134 patients. The regimen which was most accelerated included 24 patients who were treated with 3 fractions of 2 Gray $(\mathrm{Gy})(1 \mathrm{~Gy}=100 \mathrm{rads})$ per day with a treatment interval of 8 hours, to a total dose of $40 \mathrm{~Gy}$ in 20 fractions in 7 days. There was no reported increase in acute or long term toxicity. There was no difference in survival in any of the groups, and all were similar to their prior experience with conventionally delivered radiation.

More recently Keim and colleagues reported their experience with various fractionation schemes in 133 patients with glioblastomas. 10 This included a group of 38 patients who received $60 \mathrm{~Gy}$ in 16 days via 3 fractions of $1.6 \mathrm{~Gy}$ Monday to Friday and one fraction of $2.8 \mathrm{~Gy}$ on Saturdays. The paper did not mention the time interval between fractions. There was no increase in acute or long term toxicity associated with this fractionation. There was no improvement in survival as compared to their prior experience.

Whereas Simpson's study suffers from too low a total dose, Keim's study suffers from the 48 hour treatment gaps on two weekends during his fractionation scheme. Therefore, whilst neither study showed a benefit to accelerated fractionation in patients with glioblastoma, one can state that from a theoretical point of view the issue remains unresolved because of the inadequacies in both regimens. However, both these regimens represent a considerable dose in a markedly shorter period of time than is given in conventional treatment, and it is discouraging that neither showed any improvement. It remains to be seen whether a group or centre will further study accelerated fractionation in this disease, with the above shortcomings in prior studies accounted for, to finally resolve the issue.

\section{Hyperfractionation}

Hyperfractionation involves the use of a larger number of smaller fractions to a total dose which is higher than with conventional radiation. The overall treatment times of both methods are similar, and hyperfractionation usually involves the use of 2 or 3 fractions per day.

The rationale for hyperfractionation is explained by cell cycle redistribution, the difference in sensitivity to fraction size between acute and late reacting tissues, and the oxygen enhancement ratio. 1 The cells that have survived a fraction of radiation tend to be grouped in the resistant phase of the cell cycle. By increasing the number of fractions, there is a higher chance of radiating these cells in a sensitive phase as they progress through the cell cycle. However, this will hold true for epithelial cells as well as neoplastic cells, such that we would anticipate a greater degree of acute reaction. But the slow cycling cells that determine the degree of late radiation reactions are not overly affected by the larger number of fractions, such that one is able to attain a higher dose with the same degree of late radiation effect, and potentially increase the tumor control rate for the same rate of long term radiation damage. As well, late effects are more sensitive to larger fraction sizes, so that the smaller fractions used in hyperfractionation would allow an increase in total dose and a potential increase in tumor control rate for the same degree of late tissue damage. The third theoretical advantage is that there is evidence to suggest that radiation effect is less dependant on the presence of oxygen at lower fraction sizes, so that areas of hypoxia within a tumor might be less resistant to a course of hyperfractionated radiation. ${ }^{12}$

In 1982, Douglas and Worth reported an increased survival in 30 consecutive patients with glioblastoma treated with 3 fractions of $1 \mathrm{~Gy} /$ day 5 days/week to a dose of 45,54 and 60 Gy to the whole brain, with a subsequent boost of $10 \mathrm{~Gy}$ in 5-7 days by once daily fractions of 2 Gy. 13 This group was compared to 90 patients from their prior experience who had been treated from 1952 to 1974 . While this represents an interesting observation, the problems with historical control comparisons are well known (as discussed by the authors), and this study cannot be taken as evidence of efficacy of hyperfractionation.

As well, in 1982, Payne and colleagues reported the results of a randomized trial of 157 patients. ${ }^{14}$ The competing treatments were $50 \mathrm{~Gy}$ in 25 fractions once daily over 5 weeks and 36-40 Gy in 36-40 fractions given by 4 daily fractions 3 hours apart over 2 weeks. The survival curves were identical. The main criticism of this trial however lies in the fact that the hyperfractionated dose is now recognized to have been much too low, and therefore this trial has not answered the question.

The Brain Tumor Study Group (BTSG) reported a randomized trial of $110 \mathrm{cGy}$ twice a day to a total of $6600 \mathrm{cGy}$ versus 6000 cGy given at $180-200$ cGy fractions per day. 15 There was no difference in survival between the two groups.

Shin and colleagues have reported their data in 1985 from an initially randomized trial of 124 patients. 16 The patients were randomized to 1 arm of conventionally fractionated treatment, and 2 arms with hyperfractionated radiation therapy (6141 cGy/4.5 weeks/69 fractions of 89 cGy every 3 hours, 3 times/day) with or without misonidazole. The misonidazole had no significant impact on the results. However, there was a significant difference in survival favouring the hyperfractionated arms (86 patients) of the study. The problem with the interpretation of this study lies in the fact that the median survival of 29 weeks (38 patients) for the conventionally treated patients is significantly inferior to results in other large studies, and that the median survival of 39 and 49 weeks in the hyperfractionated groups is not very different from the median survival in conventionally treated patients in other studies. $2,3,14$ This group is currently treating patients to a total tumor dose of $80 \mathrm{~Gy}$ given in 80 fractions in a total treatment time of 5.5 weeks via 3 daily fractions of $1 \mathrm{~Gy}$.

Preliminary results from an RTOG randomized trial of hyperfractionation (200 patients in $3 \mathrm{arms}$ to total doses of 64.60 Gy, 72.00 Gy, and 76.80 Gy) have shown no differences between the 3 groups, with an overall median survival of 44 weeks. ${ }^{17} \mathrm{~A}$ higher dose arm of $81.60 \mathrm{~Gy}$ has now been incorporated into the study.

In summary, there is no conclusive evidence of superior results with hyperfractionated radiation therapy in the management of patients with malignant astrocytomas. However, normal tissue tolerance does not appear to have been reached, and an improvement may result from studies at higher doses which are currently underway. 


\section{BRACHYTHERAPY}

Brachytherapy involves the placement of radioactive seeds interstitially in tumors. Because of the rapid decrease in dose outside the high dose volume, there is relative sparing of adjacent normal tissues. As well, the low dose rate in brachytherapy ( $1 \mathrm{cGy} /$ minute) as compared to the dose rate in external radiation therapy (100-200 cGy/minute) is better tolerated by normal tissues, allowing a higher dose to be delivered.

This increase in local dose might be beneficial in malignant astrocytomas in view of the fact that $95 \%$ of these tumors are unifocal at presentation and $90 \%$ of tumors recur within $2 \mathrm{~cm}$ of their original location. 5,6

The current method in North America involves the stereotactic placement of either iodine-125 or irridium-92 temporarily via one or more catheters into supratentorial malignant astrocytomas. These catheters are removed after the prescribed dose has been delivered, which usually is accomplished in 5 to 7 days. Centres in both France and West Germany have been performing brachytherapy for brain tumors for 40 years, but patients with a multitude of different histologic types of brain tumors were selected and no systematic study had been undertaken to evaluate the effectiveness of this technique over conventionally managed patients. 18

Gutin and colleagues in San Francisco have the largest experience in North America with brachytherapy for patients with malignant astrocytomas. Their recent report of 95 patients in whom brachytherapy was performed for recurrence shows a median survival of 54 weeks in 45 patients with glioblastoma, and 81 weeks in 50 patients with anaplastic astrocytoma. 19

Bernstein and Laperriere have reported their preliminary results with brachytherapy. ${ }^{20}$ In 23 patients in whom brachytherapy was given in addition to external irradiation as part of initial treatment, the median survival is 60 weeks. These patients are part of a randomized study described below, and comparison with the non-implanted arm would be premature at this time. The median survival for 18 patients implanted for recurrent malignant astrocytoma is currently 44 weeks postimplant. Pathologically, $90 \%$ of both groups are glioblastoma.

As a consequence of this form of therapy, mass effect occurred in a significant number of patients over the subsequent several months. In Gutin's series, 47 of 95 patients (49\%) required reoperation subsequent to the implant for resection of varying amounts of residual tumor and gross necrotic tumor. In Bernstein's series, reoperation was performed in $26 \%$ of patients.

Currently, it would appear that brachytherapy is of possible benefit for selected patients with recurrent disease, and the role of brachytherapy as additional therapy to conventional external radiation therapy at initial presentation remains to be delineated in prospective trials ongoing in San Francisco, Toronto, and the Brain Tumor Cooperative Group (BTCG, comprises 7 major institutions in the U.S.). In all 3 studies, randomization occurs after initial surgery as deemed appropriate by the referring neurosurgeon. In San Francisco, patients are part of a randomized phase II study where the brachytherapy arm consists of $60 \mathrm{~Gy}$ delivered in a conventional manner with external radiation therapy plus an interstitial implant where $60 \mathrm{~Gy}$ would be delivered to the edge of the enhancing mass on the CT scan. Chemotherapy in the form of hydroxyurea during irradiation and
CCNU, procarbazine and vincristine will be delivered to all patients for $\mid$ year. In Toronto, patients are part of a randomized phase III study where both arms of the trial will receive $50 \mathrm{~Gy}$ in 25 fractions over 5 weeks delivered by external radiation therapy. The implant arm will receive an additional $60 \mathrm{~Gy}$ to the edge of the enhancing tumor. No chemotherapy will be delivered as part of the study. The BTCG study consists of a total $60.2 \mathrm{~Gy}$ in 35 fractions in 7 weeks to both groups. The patients randomized to the implant arm will undergo the procedure prior to their external radiation to receive an additional $60 \mathrm{~Gy}$ to the edge of the enhancing tumor. Both arms of this study will also receive intravenous BCNU every 2 months for 18 months.

\section{RADIOSENSITIZERS}

Radiosensitizers are chemicals which increase the lethal effects of radiation when administered in conjunction. Many chemicals have been found to fit this definition, however, only those that have demonstrated a potential differential effect between tumor and normal tissues would deserve further investigation. The two major classes of compounds investigated to date are hypoxic cell sensitizers and halogenated pyrimidines.

\section{Hypoxic Cell Sensitizers}

Malignant astrocytomas are often associated with large areas of necrotic tissue. At the boundary of these necrotic zones, there presumably exist tumor cells which are hypoxic. It has been well established in the laboratory that hypoxic cells are significantly more resistant to radiation than well oxygenated cells. ${ }^{21}$ Hypoxic cell sensitizers would sensitize the hypoxic cells to the effects of radiation, without increasing the effect on oxygenated cells. A differential effect would be based on hypoxic cells existing only in tumor and not in normal tissues. The most potent hypoxic cell sensitizer is oxygen, but its main disadvantage is of being rapidly metabolized by the initial hypoxic cells that it encounters, such that the concentration of oxygen decreases rapidly prior to reaching all the hypoxic cells.

In 1977, Chang reported on a pilot study of the use of hyperbaric oxygen at 3 atmospheres in 38 patients with a median survival of 38 weeks compared to 42 concurrent control patients with a median survival of 31 weeks. ${ }^{22}$ There was an escalation of dose and a variety of fractionation scheme utilized, but the important point is that the radiation parameters were the same for both groups. This was not a strictly randomized study and the patients in the hyperbaric oxygen group were slightly younger on average. Because of the small numbers, the difference was not statistically significant.

Metronidazole and misonidazole are two drugs that mimic oxygen in their ability to sensitize hypoxic cells to radiation. In addition, they have the added advantage of being able to diffuse further into hypoxic tissues without being rapidly metabolized as is the case for oxygen, thus reaching all hypoxic cells in reasonable concentrations.

In 1976, Urtasun et al reported on a small randomized study of 31 patients in which all patients received $30 \mathrm{~Gy}$ in 9 fractions delivered 3 times per week over 3 weeks. ${ }^{23}$ Half the patients received metronidazole prior to each fraction. The median survival for the metronidazole group was 26 weeks as compared to 13 weeks for the radiation alone group, and this difference was statistically significant. However, the result for the radiation 


\begin{tabular}{lccc}
\hline \multicolumn{3}{l}{ Table 1: Randomized Misonidazole Studies } & \\
\hline Author & Ref. & Year & Patients \\
\hline Bleehen et al & 25 & 1981 & 55 \\
EORTC Brain Tumour Group & 26 & 1983 & 163 \\
Stadler et al & 27 & 1984 & 45 \\
Hatlevoll et al & 28 & 1985 & 244 \\
Nelson et al & 29 & 1986 & 293 \\
Deutsch et al & 30 & 1989 & 603 \\
\hline
\end{tabular}

alone group was significantly worse than is expected for conventionally fractionated radiation and is an indication that the fractionation utilized in this study was not optimal. In a subsequent randomized study of 66 patients by the same investigators, the survival experience for conventional radiation alone was the same as for large fraction radiation with metronidazole and large fraction radiation and misonidazole. ${ }^{24}$

Subsequently, 6 further randomized trials assessing the efficacy of misonidazole in addition to either conventional or large fraction irradiation have failed to show any survival advantage (Table 1). In retrospect, these results should not be surprising. Most of the laboratory data predicting the sensitization effects were based on results achieved with single large doses of radiation, and were not based on fractionated radiation which would allow for a certain amount of reoxygenation of hypoxic areas in tumors. As well, the doses of misonidazole utilized in humans have been limited by cumulative peripheral and central neuropathy and gastrointestinal effects. As a result, the tumor concentrations achieved by this sensitizer are inadequate to demonstrate a significant clinical effect, especially when one includes the possible effect of reoxygenation during fractionated radiation. ${ }^{31}$

The latest hypoxic cell sensitizers, Ro 03-8799 (pimonidazole) and SR 2508 (etanidazole), are currently undergoing evaluation in phase I and phase II trials. ${ }^{32,33}$ The toxicity profile of these drugs allows the delivery of higher dosages in humans, with a prediction of a 5 -fold increase in effect as compared to the use of misonidazole. ${ }^{34}$ In view of their differing toxicity profiles, it has been suggested that both should be administered together as the sensitization effect is likely to be additive. ${ }^{35}$ It remains to be seen whether these new agents, either singly or in combination, will exert a significant clinical effect.

\section{Halogenated Pyrimidines}

The halogenated pyrimidines, 5-bromodeoxyuridine (BUdR) and 5-iododeoxyuridine (IUdR), are very similar to the normal DNA precursor thymidine, having a halogen substituted in place of the methyl group. As a result of this similarity, these compounds are incorporated into DNA in place of thymidine in a competitive fashion, which leads to a weakening of the DNA chain. Consequently, cells incorporating these halogenated pyrimidines are sensitized to the effects of $\mathrm{x}$-rays or ultraviolet light.

The radiobiologic rationale for the use of these compounds in malignant astrocytomas is based on the fact that only actively proliferating cells will incorporate these drugs (i.e., malignant gliomas) and not the surrounding relatively quiescent cells (normal brain).
Because these drugs are rapidly dehaligenated by the liver, early studies Japan utilized chronic intra-arterial injections of BUdR during external radiation. In 1974, Hoshino reported on 48 patients with malignant glioma treated in this fashion with $33 \%$ of patients surviving beyond 18 months in obviously selected patients. ${ }^{36}$ However, further work along these lines was suspended because of prohibitive complications associated with the indwelling arterial catheters.

Jackson and colleagues recently reported their results of four sequential phase I trials with continuous intravenous infusions of BUdR or IUdR in combination with external irradiation in 60 patients with high grade gliomas. ${ }^{37}$ They were searching for the maximal tolerable infusion doses and optimal timing relative to irradiation. The overall results were a median survival of 13 months with an 18 month survival of $24 \%$. A major difference in toxicity was the significantly lesser incidence of skin effects seen with IUdR as opposed to BUdR. In the laboratory, it has been demonstrated that IUdR and BUdR have similar sensitization effects with $x$-rays, but that IUdR has significantly less sensitization effect for ultraviolet light.

A phase I study of intra-arterial BUdR radiosensitization on 18 patients was reported by a group at Ann Arbor, Michigan. 38 The dose was escalated from $400 \mathrm{mg} / \mathrm{m}^{2} /$ day to $600 \mathrm{mg} / \mathrm{m}^{2} /$ day for 8 weeks. The estimated median survival was 22 months with 11 patients still alive, and the maximum tolerable dose was $400 \mathrm{mg} / \mathrm{m}^{2} /$ day. Ipsilateral forehead dermatitis, blepharitis, and iritis occurred in all patients, suggesting that perhaps IUdR might be a better alternative from a toxicity point of view. The intra-arterial infusion was achieved through a permanently implanted pump with a catheter placed retrograde into the external carotid artery. There were no vascular complications seen in this group.

Maximal doses of both BUdR and IUdR have been relatively well established, both by the intravenous and intra-arterial routes, for prolonged administration during external irradiation. Further studies are ongoing at a phase II level combining halogenated pyrimidines with both external irradiation and interstitial brachytherapy.

\section{HYPERTHERMIA}

In 1866, the German physician Busch described the disappearance of a facial sarcoma in a patient after a prolonged fever secondary to erysipelas. ${ }^{39}$ Since then, sporadic reports of the use of hyperthermia for various malignancies have appeared, but were largely anecdotal. There has been a significant revival of interest in this approach recently.

The rationale for the investigation of hyperthermia in brain neoplasms is based on the following considerations: ${ }^{40}$ 1) heat is cytotoxic as a single modality; 2 ) cells in S-phase (more resistant to $\mathrm{x}$-rays) are sensitive to heat; 3 ) cells in a low $\mathrm{pH}$ and hypoxic environment (resistant to $\mathrm{x}$-rays) are more sensitive to heat; 4) heat inhibits the repair of sublethal damage from $x$ rays, and therefore has a more than additive effect when combined with $\mathrm{x}$-rays; and 5) heat augments the effect of some chemotherapeutic drugs.

As can be seen from the above, hyperthermia possesses several effects which are complimentary to the effects of irradiation. As summarized by Salcman and Samaras, normal neural tissue is considered to be relatively sensitive to the effects of 
heat. ${ }^{41}$ Therefore, for a possible increase in the therapeutic index, one requires accurate homogeneous heating of the tumor volume with minimal heating of the adjacent surrounding normal brain.

Numerous reports of phase I-II studies of the use of hyperthermia alone, with radiation, and/or with chemotherapy have recently appeared in the neuro-oncological literature. ${ }^{42-49}$ Various methods of producing heat (magnetic-loop induction, interstitial microwave antennas, and radiofrequency) have been reported. The cases have been small numbers of selected patients with either recurrent or residual disease, or included as part of initial management. The thrust of the investigations thus far has been to demonstrate the feasibility and safety of single or repeated treatments with hyperthermia. It is not possible to comment on therapeutic effect at this time. A major limitation at present is the inhomogeneity of tumor volume heating, and efforts are currently underway to improve both the monitoring of intratumoral and normal brain temperatures during hyperthermia, and the homogeneity of heating. The concurrent use of interstitial microwave antennas to deliver hyperthermia during brachytherapy is a particularly attractive approach. However, information regarding any improvement in patient outcome remains to be elucidated, and would likely only be demonstrated through a multi-institutional randomized study.

\section{Particle Therapy}

Particle therapy refers to the use of sub-atomic particles as a form of treatment as opposed to photons. These particles include neutrons, protons, helium ions and heavier nuclei, and negative pi mesons (pions). The use of these particle beams offers two advantages over the use of photons: better dose localization to the tumor volume and greater biologic effect.

Particle beams (except neutrons) travel a specific depth in tissue, depending on the energy and type of particle, and deposit a large amount of their energy at the end of their path in tissue, after which there is little or no dose being delivered to deeper tissues. The specific depth at which this occurs is predictable and controllable. This phenomena is referred to as the Bragg peak. In contrast, photons (X-rays) and neutrons deposit energy in an exponentially decreasing fashion with increasing depth (see Figure 1). Thus, the Bragg peak of charged particles allows precise delivery of radiation dose to the tumor volume while excluding more normal tissue than is usually possible with photon therapy.

As well, particle beams (except proton and helium beams) have a higher biologic effect for the same dose of radiation as compared to photon beams. Subsets of tumor cells have been found to be resistant to photons because of hypoxia and because they are in the S phase of the cell cycle at the time of treatment. For most particle beams, these effects are considerably smaller. As well, there is less repair of sub-lethal or potentially lethal damage with most particle beams. This increase in biologic effect might be more damaging to normal tissues as well, and for that reason attention to normal tissue toxicity is an important aspect of these studies. Neutrons do not possess the advantage of better localization of the high dose volume, and therefore will be limited by the toxicity on normal brain, while the charged particles do have better localization of the high dose volume as compared to photons, and the intent is to restrict their additional biologic effect to the tumor volume in an effort to spare the normal brain.

\section{Fast Neutrons}

Because neutrons are not charged particles, they do not exhibit a Bragg peak, and have a distribution of dose very similar to that of photons. However, their effect is less dependant on the presence of oxygen, and therefore the rationale for their clinical use was based on possible biologic advantages only. Fast neutrons refers to neutrons that are produced at higher energies (usually in a cyclotron) than the spectrum of energies associated with neutrons in a nuclear reactor, and these latter neutrons are referred to as slow or thermal neutrons. Fast neutrons are much more penetrating in tissue than thermal neutrons, and it is for this reason that the bulk of clinical work has been performed with fast neutrons.

The initial pilot studies exploring the use of neutrons in patients with glioblastoma multiforme showed that while patients did not benefit in terms of survival, the vast majority of autopsied patients did not die of progressive tumor, but of coagulative necrosis at the site of the original tumor, with evidence of no or of very little residual tumor.50-52 These patients did die of a progressive neurologic process as a result of the necrosis induced by the neutron therapy. Very simply put, the neutron doses chosen were highly effective in controlling these brain tumors, but were above the tolerance of the brain.

With this information in mind, the Radiation Therapy Oncology Group (RTOG) entered 166 patients with glioblastoma multiforme on a randomized trial..$^{53}$ All patients received a dose of 50 Gy to their whole brain with X-rays, and were subsequently randomized to receive a boost to the tumor volume with either further X-ray therapy (15 Gy) or neutron therapy (15 Gy equivalent). There was no stastistical difference in survival between the two groups. There were autopsies performed on 24 patients in this study. Of 12 patients treated with a neutron boost, 9 had no evidence of residual or recurrent tumor in the largely necrotic mass that replaced the original tumor. This was in sharp contrast to 12 patients treated with X-ray boosts, all of whom had viable proliferating tumor masses at autopsy. The

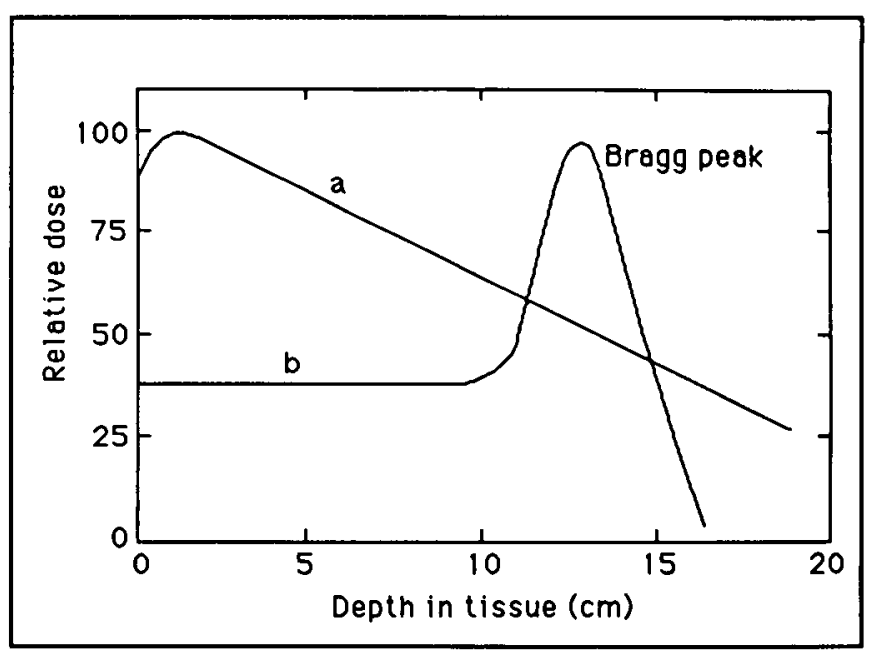

Figure I - Representative curves for the distribution of dose as a function of depth for: $a$, photon and fast neutron beams, and b, charged particle beams. 
dose of neutrons in this study was lower than in the pilot studies mixing neutrons with photons, but despite this, the dose was still above the tolerance of brain.

Duncan and colleagues published their experience with neutron therapy from Edinburgh in patients with glioblastoma. In their first study, they compared neutrons alone to photon therapy alone. ${ }^{54}$ of 17 patients who received neutron therapy, all but one patient had residual tumor at death, and 4 of 9 patients autopsied had evidence of neutron induced brain damage. There was no improvement in survival as compared to 16 patients treated with photons alone. Thus, they concluded that neutron therapy alone could not improve results in these patients. In their second study, they compared mixed-schedule (neutron/photon) radiation to photon therapy alone. ${ }^{55}$ The mixed-schedule radiation was felt to be within brain tolerance. A total of 61 patients entered this randomized study, with 31 patients receiving the mixed-schedule therapy and 30 patients receiving photon therapy alone. Once again, there was no difference in survival. On this occasion, there was no evidence of neutron induced brain damage. In their final study, they explored a novel fractionation scheme in a mixed-schedule (neutron/photon) treatment in 50 consecutive patients with malignant gliomas. 56 They found their results to be the same as with longer conventionally administered therapy.

Fast neutrons have been combined with misonidazole in a report by Kurup et al. ${ }^{57}$ Twenty-five patients were treated with neutrons and misonidazole and were compared to 44 patients treated with neutrons only. There was no difference in survival.

Finally, a study by the RTOG randomized 190 patients among 6 different neutron boost schedules. ${ }^{58}$ All patients received whole brain photon irradiation to a dose of 45 Gy at $1.5 \mathrm{~Gy}$ fractions treated 5 days per week. They were then randomized to the following neutron boosts doses: $3.6,4.2,4.8$, $5.2,5.6$ and $6.0 \mathrm{~Gy}$. There were no differences in survival between the 6 groups. Amongst the autopsied cases at all 6 neutron dose levels, there was residual viable tumor and evidence of normal brain damage at all dose levels. This would seem to clearly demonstrate a lack of a possible therapeutic window with the use of neutron therapy in the management of neoplasms of the central nervous system.

In summary, there is no demonstrated survival advantage to the use of neutrons in the management of patients with malignant astrocytomas. The studies to date have demonstrated that neutron therapy is capable of eradicating these tumors in the short term, but at a cost of brain necrosis which is incompatible with survival. Studies which have utilized a lower dose of neutron therapy which was not associated with brain necrosis, demonstrated the same survival with death occurring as a result of recurrent tumor. Suit et al agree that there has been no gain in results with neutron therapy in patients with malignant astrocytoma, but they point out that most of these studies were performed with fixed horizontal beams and/or beams with poor depth dose characteristics. 59 However, they do not include patients with malignant astrocytomas as a group to continue with further research with neutrons, and the RTOG is no longer conducting any studies with neutrons in these patients.

\section{Boron-Neutron Capture}

This technique was developed in an effort to limit the dam- aging effects of therapy to the tumor cells only with sparing of normal tissues altogether. This therapy involves the administration of a boron containing chemical which theoretically would be preferentially taken up by tumor cells as opposed to normal cells. Boron has a large neutron capture cross-section for slow or thermal neutrons, and these neutrons are present in abundance in nuclear reactors. These neutrons have an average energy of $0.025 \mathrm{eV}$, which is far less that the $10 \mathrm{eV}$ required to strip electrons from atoms and ionize tissue. When boron and these slow neutrons interact, nuclear fission occurs, with the fragments sharing $2.4 \mathrm{MeV}$ of energy in the following fashion:

$$
5^{10 \mathrm{~B}}+{ }_{0} \ln \rightarrow\left(5^{11} \mathrm{~B}\right) \rightarrow{ }_{3}^{7} \mathrm{Li}+{ }_{2}^{4} \mathrm{He}+2.4 \mathrm{MeV}
$$

The lithium and helium fragments are heavy and only travel at most $10 \mu \mathrm{m}$ from the site of the capture reaction. Therefore this reaction releases heavy fragments with sufficient energy to be highly injurious to the individual cell in which this reaction takes place. All of the other elements in tissue have a very low cross-section for neutron capture.

The actual treatment involves the injection of the boron containing compound intra-arterially and waiting 12-16 hours for the compound to clear from the vascular compartment. A craniotomy is performed and the patient must then have his head inserted into a special window into the nuclear reactor to expose his brain to thermal neutrons for a period of 30 to 90 minutes, depending on the neutron flux.

The first clinical experience was reported by Farr and Sweet and colleagues in 1954 when 10 patients with malignant brain tumors were treated. 60 The last group of patients treated in this fashion in the United States was reported by Ashbury and colleagues where 17 patients with glioblastoma and 1 patient with a medulloblastoma were treated in 1960 and 1961.61 No patient survived 1 year and the average survival was 5.7 months. At autopsy, there was evidence of brain swelling, peri-vascular fibrosis and cerebral necrosis. It was then realized that this was due to boron levels being 3 to 4 times as high in blood vessels as compared to those in tumor. All subsequent clinical work ceased in the United States after this experience.

In Japan, there has been considerable continued interest in this modality. Although most subsequent work in this area in both the U.S. and Japan has been in the laboratory, the Japanese have irradiated 77 patients with various brain tumors from 1968 to 1985.62 Forty of these patients had grade III-IV gliomas. Actuarial survival for these glioma patients was $20 \%$ at 5 years, and $10 \%$ at 10 years. The current problems with this technique are the lack of thermal neutrons of sufficient energy to penetrate to deep seated cerebral tumors, the technical problem of exposing areas other than the frontal parietal areas of the brain in the hollow made in the graphite layer of the reactor, and the diameter of the craniotomy to ascertain that all the tumor is exposed to the neutron flux.

Needless to say, this technique is extremely expensive and it is doubtful whether it will ever become an available technique to the general patient population, unless it were refined and shown to be significantly superior to current results achieved with conventional therapy, which unfortunately is currently not the case. It does however remain the most selective of all experimental therapies, and further research is certainly justified. 


\section{Pions}

This particle is particularly interesting because it has both a physical dose localization advantage (Bragg peak) and an increased biologic effect. Pions are referred to as the nuclear glue, as these particles are felt to be responsible for holding together protons and neutrons in the nucleus. Their mass is $1 / 6$ of that of a proton and 273 times that of an electron. To produce pions in a beam of sufficient intensity to be useful for clinical therapy, a very large high-intensity and high-energy proton accelerator is required. Only two such facilities are in operation in the world, and these are located in Vancouver and the SIN (The Swiss Institute for Nuclear Research) facility near Zurich. A third facility was in operation at Los Alamos, but clinical treatments ended in 1981.63

To date, most studies have been exploratory in nature, in an effort to determine the best total dose and fractionation scheme. At Los Alamos, 29 patients with malignant astrocytomas were treated with pions alone and 30 patients were treated with a mixture of pions and photons. 64 Two patients from each group were locally controlled (at 60 and 71 months for the 2 patients treated with pions alone), and all 4 patients were classified as having grade III gliomas. Only 1 patient treated with pions alone died of brain necrosis with no evidence of residual tumor at 15 months. At SIN, 20 patients with malignant gliomas have been treated (three grade III, 17 grade IV). 65 Only 1/15 patients at 9 months was free of recurrence. It would appear from both these experiences, that the doses utilized were below the level of significant tumor control probability.

In Vancouver, Goodman et al have reported on their initial experience with pions. 66 They have currently completed a pilot study with 53 patients treated in 5 different groups of dosage schemes. ${ }^{67}$ They are currently considering embarking on a randomized trial comparing their optimal pion schedule to conventional photon therapy.

To date, the clinical research with pions has focused on finding the optimal dose and fractionation. Further studies will be required before one can decide on their possible value.

\section{Charged Particles}

Currently, the only machine in the world producing heavy ions for clinical investigation is located in Berkeley. This huge and very expensive facility is able to accelerate carbon, neon, argon, and silicon ions with sufficient energy to penetrate 10$20 \mathrm{~cm}$ of tissue. As with pions, these heavy ions also have a dual advantage of greater biologic effect and greater precision in the delivery of dose to the tumor volume as compared to photons.

In 1985, Castro et al reported their early experience in a phase I-II trial in 39 patients with gliomas treated with heavy particles (helium, carbon, and neon) at Berkeley. 68 Of these, 17 had glioblastoma, 16 had anaplastic astrocytomas, and 6 patients had astrocytomas. Most patients (33/39) were treated at presentation, and 6 were treated for recurrence. There was a mixture of treatment administered, with 22 patients receiving a combination of photons and particle therapy, and 17 patients receiving particle therapy only, 6 of whom were treated for recurrence after prior photon therapy. Throughout the study, there had been a gradual escalation in the doses administered. Because these patients were treated at a physics installation, their performance status was better than average for patients with these tumors (Karnofsky performance status ranging from 70-95) by necessity. The median survival for patients with glioblastoma was 13.9 months, 7.6 months for anaplastic astrocytoma, and 24 months for lesser grade astrocytomas. These results are not significantly different from results seen in patients treated with photons. Overall, the doses used in these initial studies have resulted in tumor persistence and recurrence in most patients with minimal brain necrosis. The current studies will continue to escalate the dose and will also be done with silicone ions which offer greater biological effect than the other particles.

Austin-Seymour and colleagues have reported on the results of proton therapy in 7 patients with "intermediate to high-grade gliomas".69 All patients had received either partial or whole brain photon irradiation, with a boost to the tumor region with protons to a total median dose of 73.1 cobalt equivalent Grays. Six patients died 2 to 62 months after treatment with either persistent or locally recurrent tumor. This series is obviously too small to draw any conclusions.

\section{RADIOSURGERY}

Radiosurgery is defined as the destruction of intracranial tissue by delivering a very high dose of radiation to a small volume in the brain by stereotactic means. This is usually accomplished by a dose of several thousand cGy (range: $1600-1700 \mathrm{cGy}$ ) delivered in 1-3 fractions. The rationale is based on very accurate positioning of the high dose volume to incorporate either a small area of the brain (functional stereotactic work) or more commonly an area of abnormal tissue with a very rapid fall-off of dose outside the target lesion in the normal brain (usually in the order of several $\mathrm{mm}$ ).

Currently, there exists three different methods of accomplishing this: multiple converging cobalt beams, particle therapy, and linear accelerator techniques. The interested reader is referred to the specific articles outlining the actual techniques. ${ }^{70.74}$ Radiosurgery is by necessity restricted to a small volume of brain, with the largest volume generally being a sphere of 3 to $3.5 \mathrm{~cm}$ in diameter.

Stereotactic radiosurgery has become a frequently used modality in the management of deep seated cerebral arteriovenous malformations. ${ }^{75.76}$ It has also been utilized in ablating pituitary adenomas, acoustic neuromas, and very selected patients with recurrent solitary brain metastases. ${ }^{77.79}$ Pozza and colleagues have reported on 14 patients with low grade astrocytomas who were managed with radiosurgery alone.$^{80} \mathrm{~A}$ total dose of 16-50 Gy was delivered in either one fraction or two fractions 8 days apart. Twelve of 14 patients demonstrated a partial or complete response ( 1 patient) over a period of $11-48$ months. These were all patients with small well demarcated deep lesions. There are no reports of the use of radiosurgery in patients with malignant astrocytomas. With refinements in 3 dimensional treatment planning, it is possible that radiosurgical techniques may be useful to boost the bulky portion of malignant gliomas, but whether this will be of benefit remains to be seen.

\section{Discussion}

None of the above mentioned experimental modalities have been proven to be superior to conventionally fractionated pho- 
ton therapy in the initial management of patients with malignant astrocytomas. Except for brachytherapy, these experimental approaches have been broadly applied to these patients in a uniform fashion. Despite the fact that these patients have a universally fatal illness, we know that age, performance status, and histology are 3 prognostic factors that separate the patients into groups with different median survivals. 81 The unspoken assumption in most of these studies was that patients with these tumors were generally a homogeneous population. However, it is likely that any individual experimental form of therapy mentioned in this paper might only benefit a small subset of these patients, as different reasons for failure of treatment may be at work in different prognostic groups. What is needed are predictive assays to better characterize these tumors biologically at presentation, so that subsets of patients could be selected who might benefit from the experimental approach being investigated. Withers elegantly demonstrated how an improvement in a small proportion of patients with an experimental approach would be concealed by the larger group of patients who would not benefit. 82

It has been well demonstrated that malignant astrocytomas of the brain do infiltrate for several centimetres from the edge of the enhancing mass as demonstrated on CT scans. ${ }^{83-86}$ However, recurrence patterns are such that recurrence seems to be occurring within or immediately adjacent to the bulky portion of the tumor. It would appear that these microscopic extensions are currently not the cause of treatment failure, but may assume greater importance in future studies if the bulky portion of the disease is better controlled.

The most significant cause of treatment failure in malignant gliomas may be related to inherent radioresistance of malignant astrocytic cells. Gerweck and colleagues demonstrated that cell lines derived from malignant astrocytomas were less radiosensitive than other human tumor cell lines. ${ }^{87}$ This finding was confirmed by Fertil and Malaise in their review of published survival curves for various human tumor cell lines which demonstrated that glioblastoma was the most radioresistant tumor cell line in their comparative studies. ${ }^{88,89}$ This is related to an initial shoulder in the cell survival which is more pronounced in glioblastomas, and is indicative of a greater capacity for repair of sublethal radiation damage. This would suggest that a future area of research might focus on combining radiation with repair inhibitors.

The rapidly expanding field of molecular biology may yield important findings in gliomas in the next decade. James and colleagues have demonstrated loss of constitutional heterozygosity for loci on chromosome 10 in 28 of 29 patients with glioblastoma, whereas no similar findings were seen in 22 patients with lower grade gliomas. ${ }^{90}$ As well, loss of sequences on chromosomes 13,17 , or 22 were seen in at least one instance of each malignancy grade of adult glioma. The meaning of these observations remains to be elucidated, but would implicate loci on these chromosomes as potential sites of an active oncogene in malignant astrocytomas.

Rutka et al have demonstrated a growth factor from the medium of 8 human malignant glioma cell lines which has very similar properties to a known growth factor associated with malignancy, namely transforming growth factor. ${ }^{91}$ The actual protein has yet to be purified and identified, but further work along these lines should shed some light on the importance of this substance in the evolution and response to therapy of malignant gliomas.

\section{SUMmaRY}

There has been considerable interest in the investigation of new methods of delivering local therapy in patients with malignant astrocytomas because of the poor results with conventional treatment and because death is as a result of uncontrolled local growth of tumor. To date, there has been no improvement in results with the use of altered fractionation schemes of photon therapy, but studies are continuing at higher doses. Brachytherapy would appear to be of benefit for selected patients with recurrent disease, but the efficacy of this approach in the initial management of patients remains to be delineated in prospective randomized trials currently underway.

Misonidazole has not been shown to improve results in several randomized studies. Newer hypoxic cell sensitizers and halogenated pyrimidines are undergoing phase I-II studies and should soon be evaluated in randomized studies. Hyperthermia is currently in the developmental phase and further refinements in technology are required prior to embarking on larger clinical trials.

Fast neutron therapy has demonstrated the ability to eradicate these tumors, but only in association with brain necrosis, such that overall survival was not influenced favorably. Currently there are no active studies of neutron therapy in patients with malignant astrocytic tumors. Boron-neutron capture therapy is very attractive in theory, but further improvement in selective uptake of boron compounds by tumors and the poor depth penetrance of slow neutrons are current problems which need to be addressed. As well, this mode of therapy is extremely cumbersome and expensive, and currently, clinical studies continue only in Japan. Pion therapy has not demonstrated definite improvement in results over conventional photon therapy, but a randomized prospective trial may be undertaken in Vancouver. Currently, investigation of the use of heavy charged particles is only being performed in Berkeley, and the studies to date have been exploratory. It is hoped that further refinement of treatment techniques with newer charged particles (silicon) may yield improved results in the future.

It would appear that the single most important cause of failure in this disease may be inherent radioresistance of malignant astrocytomas based on their apparent ability to repair sub-lethal radiation injury. A future focus for investigation might include the addition of repair inhibitors to radiation in the management of this tumor once the mechanisms of repair are better understood.

\section{REFERENCES}

1. Russell DS, Rubinstein LJ. Pathology of tumours of the nervous system. Fourth edition. London: Edward Amold Lid 1977; 146282.

2. Walker MD, Alexander E, Hunt WE, et al. Evaluation of BCNU and/or radiotherapy in the treatment of anaplastic gliomas: a cooperative clinical trial. J Neurosurg 1978; 49: 333-343.

3. Walker MD, Green SB, Byar DP, et al. Randomized comparisons of radiotherapy and nitrosources for the treatment of malignant glioma after surgery. N Engl J Med 1980; 303: 1323-1329.

4. Komblith PL, Walker M. Chemotherapy for malignant gliomas. J Neurosurg 1988; 68: 1-17.

5. Hochberg FH, Pruitt A. Assumptions in the radiotherapy of glioblastoma. Neurology 1980; 30: 907-911. 
6. Bashir R, Hochberg F, Oot R. Regrowth patterns of glioblastoma multiforme related to planning of interstitial brachytherapy radiation fields. Neurosurgery 1988; 23: 27-30.

7. Choucair AK, Levin VA, Gutin PH, et al. Development of multiple lesions during radiation therapy and chemotherapy in patients with gliomas. J Neurosurg 1986; 65: 654-658.

8. Thames HD, Hendry JH. Fractionation in radiotherapy. London: Taylor \& Francis Ltd 1987; 138-169.

9. Simpson WJ, Platts ME. Fractionation study in the treatment of glioblastoma multiforme. Int J Radiat Oncol Biol Phys 1976; 1: 639-644.

10. Keim H, Potthoff PC, Schmidt K, et al. Survival and quality of life after continuous accelerated radiotherapy of glioblastoma. Radiother Oncol 1987; 9: 21-26.

11. Thames HD, Hendry JH. Fractionation in radiotherapy. London: Taylor \& Francis Ltd 1987; 53-99.

12. Palcic B, Skarsgard LD. Reduced oxygen enhancement ratio at low doses of ionizing radiation. Radiat Res 1984; 100: 328-339.

13. Douglas BG, Worth AJ. Superfractionation in glioblastoma multiforme - results of a phase II study. Int J Radiat Oncol Biol Phys 1982; 8: 1787-1794.

14. Payne DG, Simpson WJ, Keen C, et al. Malignant astrocytoma: hyperfractionated and standard radiotherapy with chemotherapy in a randomized prospective clinical trial. Cancer 1982; 50: $2301-2306$

15. Green SB, Gyar DP, Stricke TA, et al. Randomized comparisons of BCNU, streptozotocin, radiosensitizer, and fractionation in the postoperative treatment of malignant glioma (Study 7702). Proc Am Soc Clin Oncol 1984; 3: 260 (Abstract).

16. Shin $\mathrm{KH}$, Urtasun RC, Rulton D, et al. Multiple daily fractionated radiation therapy and misonidazole in the management of malignant astrocytoma: a preliminary report. Cancer $1985 ; 56: 758$ 760 .

17. Nelson DF, Urtasun RC, Saunders WM, et al. Recent and current investigations of radiation therapy of malignant gliomas. Sem Oncol 1986; 13: 46-55

18. Bernstein M. Gutin PH. Interstitial irradiation of brain tumors: a review. Neurosurgery 1981; 9: 741-750.

19. Leibel SA, Gutin PH, Wara WM, et al. Survival and quality of life after interstitial implantation of removable high-activity iodine125 sources for the treatment of patients with recurrent malignant gliomas. Int J Radiation Oncology Biol Phys 1989; 17: 1129-1139.

20. Bernstein M, Laperriere NJ, Leung P, et al. Interstitial brachytherapy for malignant brain tumors: preliminary results. Neurosurgery, in press.

21. Hall EJ. Radiobiology for the radiologist. Third edition. New York: JB Lippincott Co 1988; 179-200.

22. Chang $\mathrm{CH}$. Hyperbaric oxygen and radiation therapy in the management of glioblastoma. Natl Cancer Inst Monogr 1977; 46: 163-169.

23. Urtasun $R$, Band $P$, Chapman JD, et al. Radiation and high-dose metronidazole in supratentorial glioblastomas. $\mathrm{N}$ Engl J Med 1976; 294: 1364-1367.

24. Urtasun R, Feldstein ML, Partington J, et al. Radiation and nitroimidazoles in supratentorial high grade gliomas: a second clinical trial. Br J Cancer 1982; 46: $101-108$.

25. Bleehan NM, Wiltshire CR, Plowman PN, et al. A randomized study of misonidazole and radiotherapy for grade 3 and 4 cerebral astrocytoma. Br J Cancer 1981; 43: 436-442.

26. EORTC brain tumor group. Misonidazole in radiotherapy of supratentorial malignant brain gliomas in adult patients: a randomized double-blind study. Eur J Cancer Clin Oncol 1983; 19: 39-42.

27. Stadler B, Karcher KH, Kogelnik HD, et al. Misonidazole and irradiation in the treatment of high-grade astrocytomas: further report of the Vienna study group. Int J Radiation Oncology Biol Phys 1984; 10: 1713-1717.

28. Hatlevoll R, Lindegaard K, Hagen S, et al. Combined modality treatment of operated astrocytomas grade 3 and 4 . A prospective and randomized study of misonidazole and radiotherapy with two different radiation schedules and subsequent CCNU chemotherapy. Stage II of a prospective multicenter trial of the
Scandinavian Glioblastoma Study Group. Cancer 1985; 56: 41 . 47.

29. Nelson DF, Diener-West $M$, Weinstein AS, et al. A randomized comparison of misonidazole sensitized radiotherapy plus BCNU and radiotherapy plus BCNU for treatment of malignant glioma after surgery: final report of an RTOG study. Int J Radiation Oncology Biol Phys 1986; 12: 1793-1800.

30. Deutsch M, Green SB, Strike TA, et al. Results of a randomized trial comparing BCNU plus radiotherapy, streptozotocin plus radiotherapy, $\mathrm{BCNU}$ plus hyperfractionated radiotherapy, and $\mathrm{BCNU}$ following misonidazole plus radiotherapy in the postoperative treatment of malignant glioma. Int J Radiation Oncology Biol Phys 1989; 16: 1389-1396.

31. Brown JM. Clinical trials of radiosensitizers: what should we expect? Int J Radiation Oncology Biol Phys 1984; 10: 425-429.

32. Coleman CN, Wasserman TH, Urtasun RC, et al. Phase I trial of the hypoxic cell radiosensitizer SR-2508: the results of the five to six week drug schedule. Int J Radiation Oncology Biol Phys 1986; 12: 1105-1108

33. Newman HFV, Bleehen NM, Ward R, et al. Hypoxic cell radiosensitizers in the treatment of high grade gliomas: a new direction using combined RO 03-8799 (pimonidazole) and SR 2508 (etanidazole). Int J Radiation Oncology Biol Phys 1988; 15: 677 684.

34. Dische S, Saunders MI, Dunphy EP, et al. Concentrations achieved in human tumors after administration of misonidazole, SR-2508 and RO 03-8799. Int J Radiation Oncology Biol Phys 1986; 12 : 1109-1111.

35. Newman HFV, Bleeken NM, Workman P. A phase I study of the combination of two hypoxic cell radiosensitizers, RO 03-8799 and SR-2508: toxicity and pharmacokinetics. Int J Radiation Oncology Biol Phys 1986; 12: 1113-1116.

36. Hoshino T. Radiosensitization of brain tumours. In: Deely TJ, ed. Modern Radiotherapy and Oncology. London: Butterworths $1974 ; 170-183$.

37. Jackson D, Kinsella T, Rowland J, et al. Halogenated pyrimidines as radiosensitizers in the treatment of glioblastoma multiforme Am J Clin Oncol (CCT) 1987; 10: 437-443.

38. Greenberg HS, Chandler WF, Diaz RF, et al. Intra-arterial bromodeoxyuridine radiosensitization and radiation in treatment of malignant astrocytomas. J Neurosurg 1988; 69: 500-505.

39. Busch W. Uber den Einfluss, welchen heftigere erysipein zuweillen auf organisierte neubildunge ausuben. Verhandlungen des naturlistorischen Vereines der preussischen Rheinlande und Westphalens 1866; 23: 28.

40. Hall EJ. Radiobiology for the Radiologist. Third edition. New York: JB Lippincott Co 1988; 293-330.

41. Salcman M, Samaras GM. Hyperthermia for brain tumors: biophysical rationale. Neurosurgery 1981; 9: 327-335.

42. Silberman AW, Rand RW, Storm FK, et al. Phase I trial of thermochemotherapy for brain malignancy. Cancer 1985; 56: 48-56.

43. Silberman AW, Rand RW, Krag DN, et al. Effect of localized magnetic-induction hyperthermia on the brain. Cancer 1986; 57 $1401-1404$.

44. Wen HL. Dahele JS, Mehal ZD, et al. Application of invasive microwave hyperthermia for the treatment of gliomas. J Neurooncol 1988; 6: 93-101.

45. Tanaka R, Kim CH, Yamada N, et al. Radiofrequency hyperthermia for malignant brain tumors: preliminary results of clinical trials. Neurosurgery 1987; 21: 478-483.

46. Roberts DW, Strohbehn JW, Coughlin CT, et al. Iridium-192 brachytherapy in combination with interstitial microwaveinduced hyperthermia for malignant glioma. App. Neurophysiol 1987; 50: 287-291.

47. Roberts DW, Coughlin CT, Wong TZ, et al. Interstitial hyperthermia and iridium brachytherapy in treatment of malignant glioma. J Neurosurg 1986; 64: 581-587.

48. Salcman M, Samaras GM. Interstitial microwave hyperthermia for brain tumors. J Neurooncol 1983; 1: 225-236.

49. Winter A, Laing J. Paglione R, et al. Microwave hyperthermia for brain tumors. Neurosurgery $1985 ; 17-387-399$. 
50. Parker RG, Berry HC, Gerdes AJ, et al. Fast neutron beam radiotherapy of glioblastoma multiforme. Am J Roentgenol 1976; 127: $331-335$

51. Laramore GE, Griffin TW, Gerdes AJ, et al. Fast neutron and mixed (neutron/photon) beam teletherapy for grades III and IV astrocytomas. Cancer 1978; 42: 96-103.

52. Catterall M, Bloom JG, Ash DV, et al. Fast neutrons compared with megavoltage $X$-rays in the treatment of patients with supratentorial glioblastoma: a controlled pilot study. Int J Radiat Oncol Biol Phy 1980; 6: 261-266.

53. Griffin TW, Davis R, Laramore $G$, et al. Fast neutron radiation therapy for glioblastoma multiforme. Am J Clin Oncol 1983; 6: 661-667.

54. Duncan W, McLelland J. Jack WJL, et al. Report of a randomized pilot study of the treatment of patients with supratentorial gliomas using neutron irradiation. Br J Radiol 1986; 59: 373377.

55. Duncan W, McLelland J, Jack WJL, et al. The results of a randomized trial of mixed-schedule (neutron/photon) irradiation in the treatment of supratentorial grade III and grade IV astrocytoma. Br J Radiol 1986; 59: 379-383.

56. Duncan W, McLelland J, Davey P, et al. A phase I study of mixed (neutron/photon) irradiation using two fractions per day in the treatment of high-grade astrocytomas. Br J Radiol 1986; 59: 441-444.

57. Kurup PD, Pajak TF, Hendrickson FR, et al. Fast neutrons and misonidazole for malignant astrocytomas. Int $J$ Radiat Oncol Biol Phys 1985; 11: 679-686.

58. Laramore GE, Diener-West M. Griffin TW, et al. Randomized neutron dose searching study for malignant gliomas of the brain: results of an RTOG study. Int J Radiat Oncol Biol Phys 1988; 14: 1093-1102.

59. Suit HD, Griffin TW, Castro JR, et al. Particle radiation therapy research plan. Am J Clin Oncol 1988; 11: 330-341.

60. Farr LE, Sweet WH, Robertson JS, et al. Neutron capture therapy with boron in the treatment of glioblastoma multiforme. Am J Roentgenol 1954; 71 : 279-293.

61. Asbury AK, Ojemann RG, Nielsen SL, et al. Neuropathologic study of fourteen cases of malignant brain tumor treated by boron-10 slow neutron capture radiation. J Neuropathol Exp Neurol 1972; 31: 278-303

62. Hatanaka H. Introduction. In: Hanataka H, ed. Boron-Neutron Capture Therapy For Tumors. Japan: Nishimura Co Ltd 1986; 1 28.

63. Hall EJ. Radiobiology for the radiology. Third edition. New York: JB Lippincott Co 1988; 261-292.

64. von Essen CF, Bagshaw MA, Bush SE, et al. Long-term results of pion therapy at Los Alamos. Int J Radiat Oncol Biol Phys 1987; 13: 1389-1398.

65. Schmitt G, von Essen CF, Greiner R, et al. Review of the SIN and Los Alamos pion trials. Radiat Res 1985; 104: S-272-S-278.

66. Goodman GB, Dixon P, Lam GKY, et al. Preparatory clinical studies of pi-mesons at TRIUMF. Radiat Res 1985; 104: S-279-S284.

67. Goodman GB, personal communication 1987

68. Castro JR, Saunders WM, Austin-Seymour MM, et al. A phase I-II trial of heavy charged particle irradiation of malignant glioma of the brain: a Northern California Oncology Group study. Int J Radiat Oncol Biol Phys 1985; 11: 1795-1800.

69. Austin-Seymour M, Munzenrider JE, Goitein M, et al. Progress in low-LET heavy particle therapy: intracranial and paracranial tumors and uveal melanomas. Radiat Res 1985; 104: S219-S226.

70. Leksell L. Stereotactic radiosurgery. J Neurol Neurosurg Psychiatry 1983; 46: 797-803.
71. Bradshaw JD. Special report. The stereotactic radiosurgery unit in Sheffield. Clinical Radiology 1986; 37: 277-279.

72. Fabrikant JI, Lyman JT, Hosobuchi Y. Stereotactic heavy-ion Bragg peak radiosurgery for intra-cranial vascular disorders: method for treatment of deep arteriovenous malformations. $\mathrm{Br} \mathrm{J}$ Radiol 1984; 57: 479-490.

73. Colombo F, Benedetti A, Pozza F, et al. External stereotactic irradiation by linear accelerator. Neurosurgery 1985; 16: 154-160.

74. Podgorsak EB, Olivier A, Pla M, et al. Dynamic stereotactic radiosurgery. Int J Radiation Oncology Biol Phys 1988; 14: 115-126.

75. Kjellberg RN. Stereotactic Bragg peak proton beam radiosurgery for cerebral arteriovenous malformations. Ann Clin Res 1986; 18 Suppl 47: 17-19.

76. Fabrikant JI, Lyman JT, Frankel KA. Heavy charged-particle Bragg peak radiosurgery for intracranial vascular disorders. Radiat Res 1985; 104: S244-S258.

77. Degerblad M, Rahn T, Bergstrand G, et al. Long-term results of stereotactic radiosurgery to the pituitary gland in Cushing's disease. Acta Endocrinologica 1986; 112: 310-314.

78. Noren G. Arndt J, Hindmarsh T. Stereotactic radiosurgery in cases of acoustic neurinoma: further experiences. Neurosurgery 1983; 13: 12-22.

79. Sturm V, Kober B, Hover KH, et al. Stereotactic percutaneous single dose irradiation of brain metastases with a linear accelerator. Int J Radiation Oncology Biol Phys 1987; 13: 279-282.

80. Pozza F, Colombo F, Chierego G, et al. Low-grade astrocytomas: treatment with unconventionally fractionated external beam stereotactic radiation therapy. Radiology 1989; 171: 565-569.

81. Shapiro WR. Therapy of adult malignant brain tumors: what have the clinical trials taught us? Sem Oncol 1986; 13: 38-45.

82. Withers HR. Neutrons and other clinical trials: impossible dreams? Int J Radiat Oncol Biol Phys 1987; 13: 1967-1970.

83. Burger PC, Heinz ER, Shibata T, et al. Topographic anatomy and CT correlations in the untreated glioblastoma multiforme. App Neurophysiol 1983, 46: 698-704.

84. Halperin EC, Bentel G, Heinz ER, et al. Radiation therapy treatment planning in supratentorial glioblastoma multiforme: an analysis based on post mortem topographic anatomy with CT correlations. Int J Radiation Oncology Biol Phys 1989; 17; 1347 1350.

85. Halperin EC, Burger PC, Bullard DE. The fallacy of the localized supratentorial malignant glioma. Int J Radiat Oncol Biol Phys 1988; 15: 505-509.

86. Kelly PJ, Daumas-Duport C, Kispert DB, et al. Imaging-based stereotaxic serial biopsies in untreated intracranial glial neoplasms. J Neurosurg 1987; 66: 865-874.

87. Gerweck LE, Komblith PL, Burlett BS, et al. Radiation sensitivity of cultured human glioblastoma cells. Radiology 1977; 125: $231-234$

88. Fertil B, Malaise EP. Intrinsic radiosensitivity of human cell lines is correlated with radioresponsiveness of human tumors: analysis of 101 published survival curves. Int J Radiation Oncology Biol Phys 1985; 11: 1699-1707.

89. Malaise EP, Fertil B, Chavaudra N, et al. Distribution of radiation sensitivities for human tumor cells of specific histological types: comparison of in vitro to in vivo data. Int J Radiation Oncology Biol Phys 1986; 12: 617-624.

90. James CD, Carlbom E, Dumanski JP, et al. Clonal genomic alterations in glioma malignancy stages. Cancer Research 1988; 48 5546-5551.

91. Rutka JT, Rosenblum ML, Stern R, et al. Isolation and partial purification of growth factors with TGF-like activity from human malignant gliomas. J Neurosurg 1989; 71: 875-883. 